VOLUMe 27 (2020) 194-205

DOI: $10.24330 /$ ieja.663066

\title{
EXAMPLES OF (NON-)BRAIDED TENSOR CATEGORIES
}

\author{
Mejía Castaño Adriana \\ Received: 11 April 2019; Revised: 23 July 2019; Accepted: 24 July 2019 \\ Communicated by Abdullah Harmancı
}

\begin{abstract}
Six examples of non-braidable tensor categories which are extensions of the category $\operatorname{Comod}(H)$, for $H$ a supergroup algebra; and two examples of braided categories where the only possible braiding is the trivial braiding are introduced.
\end{abstract}

Mathematics Subject Classification (2010): 18D10

Keywords: Supergroup algebra, braidings, tensor category

\section{Introduction}

Braided categories were introduced by Joyal and Street [5]. They are related to knot invariants, topology and quantum groups, since they can express symmetries. Some examples of braided categories are:

- graded modules over a commutative ring,

- (co)modules over a (co)quasi-triangular Hopf algebra,

- the Braid category, [5, Section 2.2],

- the center of a tensor category.

In the last example, we begin with a tensor category and construct a braided one. In a general scenario, a natural question is it is possible to construct braidings starting with tensor categories. In particular, if $G$ is a finite group, can a $G$-extension of a tensor category be braided? In this work we show that this can be done in very few cases. Then, an extension of a braided category is not necessarily braided, so it is really complicated to extend that property.

However, constructing examples of non-braided categories is also important. A big family of these come from the category of (co)modules of a Hopf algebra without a (co)quasi-triangular structure, see [9, T 10.4.2]. Masuoka in [6] and [7] constructs explicit examples of non-Quasi-triangular or non-CoQuasi-triangular Hopf algebras. In particular these Hopf algebras can not be obtained from any group algebra by twist (or cocycle) deformation. Other examples were constructed in [4]. 
In the literature there are a few explicit examples of tensor categories, for this reason we construct in [8] eight tensor categories, following the description introduced in [3] of Crossed Products. These categories extend the module category over certain quantum groups, called supergroup algebras. In a few words, a crossed product tensor category is, as Abelian category, the direct sum of copies of a fixed tensor category, and the tensor product comes from certain data. Then founding all possible data, we explicitly construct tensor categories.

In the same work [3], the author also describes all possible braidings over a crossed product. Following this, three conditions were introduced to decide if a $G$-crossed product is braidable:

(1) the base category has to be braided,

(2) $G$ has to be Abelian, and the biGalois objects associated to each crossed product have to be trivial,

(3) the 3-cocycle associated to each crossed product over an specific supergroup algebra has to be trivial, if $G$ is the cyclic group of order 2 .

The goal in the present paper is to obtain all possible braidings over the categories introduced in [8]. With this, only two categories of the eight found in [8] are braided with the trivial braiding only, and the other 6 are not braidable.

In [8, Theorem 6.3], using the Frobenius-Perron dimension, we proved that these eight categories are the module category of a quasi-Hopf algebra. Although we do not know how to explicitly compute these algebras, as a corollary of this work, we know that six of these algebras are non-Quasi-triangular and two are Quasitriangular only. In particular, we are obtaining information about certain quasiHopf algebras without knowing them explicitly; showing how useful it is to work in the category world. In a future, when we can explicitly describe these quasi-Hopf algebras, we will already know how their Quasi-triangular structures are.

\section{Preliminaries and notation}

Throughout this paper we shall work over an algebraically closed field $\mathbb{k}$ of characteristic zero. For basic knowledge of Hopf algebras see [9]. Let $H$ be a finitedimensional Hopf algebra and $A$ be a left $H$-comodule. Then $A$ is also a right $H$-comodule with right coaction $a \mapsto a_{0} \otimes S\left(a_{-1}\right)$, see [1, Proposition 2.2.1(iii)]. A left $H$-Galois extension of $A^{c o(H)}$ is a left $H$-comodule algebra $(A, \rho)$ such that $A \otimes_{A^{c o(H)}} A \rightarrow H \otimes A, a \otimes b \mapsto(1 \otimes a) \rho(b)$ is bijective. Similarly, we define right $H$-Galois extension. 
Consider $L$ another finite-dimensional Hopf algebra. An (H,L)-biGalois object [10] is an algebra $A$ that is a left $H$-Galois extension and a right $L$-Galois extension of the base field $\mathbb{k}$ such that the two comodule structures make it an $(H, L)$ bicomodule. Two biGalois objects are isomorphic if there exists a bijective bicomodule morphism that is also an algebra map. For $A$ an $(H, L)$-biGalois object, define the tensor functor

$$
\mathcal{F}_{A}: \operatorname{Comod}(L) \rightarrow \operatorname{Comod}(H), \quad \mathcal{F}_{A}=A \square_{L}-.
$$

By [10], every tensor functor between comodule categories is one of these, and $\mathcal{F}_{A} \simeq \mathcal{F}_{B}$ as tensor functors if and only if $A \simeq B$ as biGalois objects.

If $A=H$, then every natural monoidal equivalence $\beta: \mathcal{F}_{H} \rightarrow \mathcal{F}_{H}$ is given by

$$
f \otimes \operatorname{id}_{X}: H \square_{H} X \rightarrow H \square_{H} X, \quad\left(X, \rho_{X}\right) \in \operatorname{Comod}(H),
$$

where $f: H \rightarrow H$ is a bicomodule algebra isomorphism.

Lemma 2.1. Every natural monoidal equivalence $\operatorname{id}_{\operatorname{Comod}(H)} \rightarrow \operatorname{id}_{\operatorname{Comod}(H)}$ is given by $\left(\varepsilon f \otimes \operatorname{id}_{X}\right) \rho_{X}$.

Proof. For $X \in \operatorname{Comod}(H)$, the coaction induces an isomorphism $X \simeq H \square_{H} X$ with inverse induced by $\varepsilon$, the counit. Then $\operatorname{id}_{\operatorname{Comod}(H)} \simeq \mathcal{F}_{H}$ as tensor functors. Since all natural monoidal autoequivalences of $\mathcal{F}_{H}$ are given by $f \otimes \operatorname{id}_{X}$ then all natural monoidal autoequivalences of $\operatorname{id}_{\operatorname{Comod}(H)}$ are given by $\left(\varepsilon f \otimes \operatorname{id}_{X}\right) \rho_{X}$.

Definition 2.2. [9, Definition 10.1.5] $(H, R)$ is a Quasi-triangular (or QT) Hopf algebra if $H$ is a Hopf algebra and there exists $R \in H \otimes H$, called the $R$-matrix, invertible such that

$$
(\Delta \otimes \mathrm{id}) R=R^{13} R^{23}, \quad(\mathrm{id} \otimes \Delta) R=R^{13} R^{12}, \quad \Delta^{o p}(h)=R \Delta(h) R^{-1}, h \in H .
$$

Dualizing we can define, $(H, r)$ is a CoQuasi-triangular (or CQT) Hopf algebra if $H$ is a Hopf algebra and $r: H \otimes H \rightarrow \mathbb{k}$, called the $r$-form, is a linear functional which is invertible with respect to the convolution multiplication and satisfies for arbitrary $a, b, c \in H$

$$
\begin{gathered}
r(c \otimes a b)=r\left(c_{1} \otimes b\right) r\left(c_{2} \otimes a\right), \quad r(a b \otimes c)=r\left(a \otimes c_{1}\right) r\left(b \otimes c_{2}\right), \\
r\left(a_{1} \otimes b_{1}\right) a_{2} b_{2}=r\left(a_{2} \otimes b_{2}\right) b_{1} a_{1} .
\end{gathered}
$$

Remark 2.3. Drinfeld defined a quantum group as a non-commutative, noncocommutative Hopf algebra. Examples of these are the QT Hopf algebras. The 
importance of quantum groups lies in they allow to construct solutions for the quantum Yang-Baxter equation in statistical mechanics (the $R$-matrix is a solution of this equation). An example of quantum group are the supergroup algebras.

A supergroup algebra is a supercocommutative Hopf algebra of the form $\mathbb{k}[G] \ltimes$ $\wedge V$, where $G$ is a finite group and $V$ is a finite-dimensional $G$-module. They appear and have an interesting role in the classification of triangular algebras, see [2, Theorem 4.3].

Example 2.4. Consider $H=\mathbb{k} C_{2} \ltimes \mathbb{k} V$, for $V$ a 2-dimensional vector space and $C_{2}$ the 2-cyclic group generated by $u$ with $u \cdot v=-v$ for $v \in V$. As an algebra, it is generated by elements $v \in V, g \in C_{2}$ subject to relations $v w+w v=0 ; g v=(g \cdot v) g$ for all $v, w \in V, g \in C_{2}$. The coproduct and antipode are determined by

$$
\Delta(v)=v \otimes 1+u \otimes v ; \Delta(g)=g \otimes g ; S(v)=-u v ; S(g)=g^{-1}, \quad v \in V, g \in C_{2} .
$$

Taking $R=\frac{1}{2}(1 \otimes 1+1 \otimes u+\otimes 1-u \otimes u),(H, R)$ is a QT-Hopf algebra. We can construct a CoQuasi-triangular structure taking $r=R^{*}$ since $H$ is auto-dual. Then $\left(H, R^{*}\right)$ is a CQT-Hopf algebra.

Definition 2.5. A finite tensor category is a locally finite, 7-linear, rigid, monoidal Abelian category $\mathcal{D}$ with $\operatorname{End}_{\mathcal{D}}(\mathbf{1}) \cong 7$. Given a finite group $\Gamma$, a (faithful) $\Gamma$ grading on a finite tensor category $\mathcal{D}$ is a decomposition $\mathcal{D}=\oplus_{g \in \Gamma} \mathcal{D}_{g}$, where $\mathcal{D}_{g}$ are full Abelian subcategories of $\mathcal{D}$ such that

- $\mathcal{D}_{g} \neq 0$

- $\otimes: \mathcal{D}_{g} \times \mathcal{D}_{h} \rightarrow \mathcal{D}_{g h}$ for all $g, h \in \Gamma$.

We have that $\mathcal{C}:=\mathcal{D}_{e}$ is a tensor subcategory of $\mathcal{D}$. The category $\mathcal{D}$ is call a $\Gamma$-extension of $\mathcal{C}$. Denote by $[V, g]$ the homogeneous elements in $\mathcal{D}$, for $V \in \mathcal{D}_{g}$, $g \in \Gamma$.

A braided tensor category is a tensor category $\mathcal{C}$ with natural isomorphisms $c_{X, Y}: X \otimes Y \rightarrow Y \otimes X$ such that

$$
\begin{aligned}
& \alpha_{V, W, U} c_{U, V \otimes W} \alpha_{U, V, W}=\left(\operatorname{id} \otimes c_{U, W}\right) \alpha_{V, U, W}\left(c_{U, V} \otimes \mathrm{id}\right), \\
& \alpha_{W, U, V}^{-1} c_{U \otimes V, W} \alpha_{U, V, W}^{-1}=\left(c_{U, W} \otimes \mathrm{id}\right) \alpha_{U, W, V}^{-1}\left(\mathrm{id} \otimes c_{V, W}\right) .
\end{aligned}
$$

If $(H, r)$ is a CQT-Hopf algebra then $\operatorname{Comod}(H)$ is a braided tensor category with braiding given by $c_{V \otimes W}(x \otimes y)=r\left(y_{-1} \otimes x_{-1}\right) y_{0} \otimes x_{0}$, for all $V, W \in \operatorname{Comod}(H)$.

The following theorem gives us the first condition to know when an extension can be braided. 
Theorem 2.6. Let $\mathcal{D}=\oplus_{g \in G} \mathcal{D}_{g}$ be a $\Gamma$-extension of $\mathcal{C}$. If $\mathcal{D}$ is a braided tensor category then $\mathcal{C}$ is a braided tensor category.

Proof. Let $c$ be the braiding of $\mathcal{D}$, then $c_{[V, e],[W, e]}:[V \otimes W, e] \rightarrow[W \otimes V, e]$ and $c_{[V, e],[W, e]}=\left[\bar{c}_{V, W}, e\right]$ for some natural isomorphism $\bar{c}_{V, W}: V \otimes W \rightarrow W \otimes V$, for $V, W$ objects in $\mathcal{C}$. Since the associativity isomorphism satisfies $a_{[V, e],[W, e],[U, e]}=$ $\left[\bar{a}_{V, W, U}, e\right]$, where $\bar{a}$ is the associativity morphism for $\mathcal{C}$; then $\bar{c}$ is a braiding for $\mathcal{C}$.

In [3], the author describes and classifies a family of such extensions and calls it crossed product tensor category. Fix $H$ a finite-dimensional Hopf algebra. In the case when $\mathcal{C}=\operatorname{Comod}(H)$, in [8], we described crossed products in terms of Hopf-algebraic datum. A continuation they are introduced.

If $g \in G(H)$ and $L$ is a $(H, H)$-biGalois object then the cotensor product $L \square_{H} \mathbb{k}_{g}$ is one-dimensional. Let $\phi(L, g) \in \Gamma$ be the group-like element such that $L \square_{H} \mathbb{k}_{g} \simeq$ $\mathbb{k}_{\phi(L, g)}$ as left $H$-comodules. Assume that $A$ is an $H$-biGalois object with left $H$ comodule structure $\lambda: A \rightarrow H \otimes_{\mathbb{k}} A$. If $g \in G(H)$ is a group-like element we can define a new $H$-biGalois object $A^{g}$ on the same underlying algebra $A$ with unchanged right comodule structure and a new left $H$-comodule structure given by $\lambda^{g}: A^{g} \rightarrow H \otimes_{\mathbb{k}} A^{g}, \lambda^{g}(a)=g^{-1} a_{-1} g \otimes a_{0}$ for all $a \in A$.

Theorem 2.7. [8, Lemma 5.7, Theorem 5.4] Let $\Upsilon=\left(L_{a},\left(g(a, b), f^{a, b}\right), \gamma\right)_{a, b \in \Gamma} b e$ a collection where

- $L_{a}$ is a $(H, H)$-biGalois object;

- $g(a, b) \in G(H)$;

- $f^{a, b}:\left(L_{a} \square_{H} L_{b}\right)^{g(a, b)} \rightarrow L_{a b}$ are bicomodule algebra isomorphisms;

- $\gamma \in Z^{3}\left(G(H), \mathbb{k}^{\times}\right)$normalized,

such that for all $a, b, c \in \Gamma$ :

$$
\begin{gathered}
L_{e}=H, \quad\left(g(e, a), f^{e, a}\right)=\left(e, \operatorname{id}_{L_{a}}\right)=\left(g(a, e), f^{a, e}\right) ; \\
\phi\left(L_{a}, g(b, c)\right) g(a, b c)=g(a, b) g(a b, c) ; \\
f^{a b, c}\left(f^{a, b} \otimes \operatorname{id}_{L_{c}}\right)=f^{a, b c}\left(\operatorname{id}_{L_{a}} \otimes f^{b, c}\right) .
\end{gathered}
$$

Then $\operatorname{Comod}(H)(\Upsilon):=\oplus_{g \in \Gamma} \operatorname{Comod}(H)$ as a structure of tensor category. 
Proof. We give an sketch of the proof. Let $\Upsilon$ be a collection as in the Theorem. For $V, W \in \operatorname{Comod}(H), a, b \in \Gamma$, define

$$
\begin{aligned}
{[V, a] \otimes[W, b] } & :=\left[V \otimes\left(L_{a} \square_{H} W\right) \otimes \mathbb{k}_{g(a, b)}, a b\right], \\
{[V, 1]^{*}: } & =\left[V^{*}, 1\right], \\
{[\mathbb{k}, a]^{*} } & :=\left[\mathbb{k}_{g\left(a, a^{-1}\right)}, a^{-1}\right] .
\end{aligned}
$$

Using [8, Eq (5.8)], we obtain the pentagon diagram and therefore $\operatorname{Comod}(H)(\Upsilon)$ is a monoidal category. Since $\operatorname{Comod}(H)$ is finite tensor category, then $\operatorname{Comod}(H)(\Upsilon)$ is also finite tensor category.

The following theorem gives us a second condition to decided if our extensions can be braided.

Theorem 2.8. If $\operatorname{Comod}(H)(\Upsilon)$ is braided with braiding $c$ then the following conditions have to hold

(1) $L_{a} \simeq H$ for all $a \in \Gamma$,

(2) $\Gamma$ is Abelian,

(3) $\Upsilon$ comes from a data $\left(g, f^{a, b}, \gamma\right)_{a, b \in \Gamma}$ with

- $g \in Z^{2}(\Gamma, G(H))$ normalized,

- $f^{a, b}: H^{g(a, b)} \rightarrow H$ a bicomodule algebra isomorphism with $f^{a b, c} f^{a, b}=$ $f^{a, b c} f^{b, c}$,

- $\gamma \in Z^{3}\left(G(H), \mathbb{k}^{\times}\right)$normalized.

Proof. (1) Take, for any $V \in \operatorname{Comod}(H), c_{[V, e][1, a]}:[V, a] \rightarrow\left[L_{a} \square_{H} V\right.$,a], this defines a natural isomorphism $\bar{c}_{a}: \operatorname{id}_{\mathcal{C}} \rightarrow L_{a} \square_{H}$ - which is monoidal since $c$ is a braiding. Then $L_{a} \simeq H$ as bicomodule algebras for all $a \in \Gamma$.

(2) Consider $c_{[\mathbf{1}, a][\mathbf{1}, b]}:\left[\mathbb{k}_{g(a, b)}, a b\right] \rightarrow\left[\mathbb{k}_{g(b, a)}, b a\right]$ then $a b=b a$ for all $a, b \in \Gamma$ and $\Gamma$ is Abelian.

(3) Since $L_{a}$ is trivial, then Equation (4) of Theorem 2.7 is equivalent to $g \in$ $Z^{2}(\Gamma, G(H))$ and it is normalized by Equation (3) of Theorem 2.7. Moreover $f^{a, b}$ : $H^{g(a, b)} \rightarrow H$ is a bicomodule algebra isomorphism that satisfies $f^{a b, c} f^{a, b}=f^{a, b c} f^{b, c}$ which is equivalent to Equation (5) of Theorem 2.7.

Remark 2.9. By definition of bicomodule morphism, $f^{a, b}: H \rightarrow H$ has to be an algebra isomorphism such that $f^{a, b}(h)_{1} \otimes f^{a, b}(h)_{2}=g^{-1} h_{1} g \otimes f^{a, b}\left(h_{2}\right)$ and $f^{a, b}(h)_{1} \otimes f^{a, b}(h)_{2}=f^{a, b}\left(h_{1}\right) \otimes h_{2}$, then $g^{-1} h_{1} g \otimes f^{a, b}\left(h_{2}\right)=f^{a, b}\left(h_{1}\right) \otimes h_{2}$. 
In the case when $H=\wedge V \# \mathbb{k} C_{2}$, as Example 2.4, using the previous Theorem we obtained eight tensor categories non-equivalent pairwise, [8, Section 6.3], named $\mathcal{C}_{0}(1, \mathrm{id}, \pm 1), \mathcal{C}_{0}(u, \iota, \pm 1), \mathcal{D}(1, \mathrm{id}, \pm 1), \mathcal{D}(u, \iota, \pm 1)$.

In all cases, the underlying Abelian category is $\operatorname{Comod}(H) \oplus \operatorname{Comod}(H)$ and for $V, W, Z \in \operatorname{Comod}(H)$ they are defined in the following way:

- The tensor product, dual objects and associativity in $\mathcal{C}_{0}(1, \mathrm{id}, \pm 1)$ are given by

$$
\begin{aligned}
{[V, e][W, g] } & =[V \otimes W, g], & {[V, u][W, g] } & =\left[V \otimes \mathbf{U}_{0} \square_{H} W, u g\right], \\
{[V, e]^{*} } & =\left[V^{*}, e\right], & {[\mathbf{1}, u]^{*} } & =[\mathbb{k}, u],
\end{aligned}
$$

$\alpha_{[V, u],[W, u],[Z, u]}$ is not trivial, and $\mathbf{U}_{0}$ is certain BiGalois object, see [8, Section 4].

- The tensor product, dual objects and associativity in $\mathcal{C}_{0}(u, \iota, \pm 1)$ are given by

$$
\begin{aligned}
{[V, e][W, e] } & =[V \otimes W, 1], & {[V, u][W, u] } & =\left[V \otimes \mathbf{U}_{0} \square_{H} W \otimes \mathbb{k}_{u}, e\right], \\
{[V, e][W, u] } & =[V \otimes W, u], & {[V, u][W, e] } & =\left[V \otimes \mathbf{U}_{0} \square_{H} W, u\right], \\
{[V, e]^{*} } & =\left[V^{*}, e\right], & {[\mathbf{1}, u]^{*} } & =\left[\mathbb{k}_{u}, u\right],
\end{aligned}
$$

$\alpha_{[V, u],[W, u],[Z, u]}$ is not trivial.

- The tensor product, dual objects and associativity in $\mathcal{D}(1, \mathrm{id}, \pm 1)$ are given by

$$
\begin{aligned}
{[V, e][W, g] } & =[V \otimes W, g], & {[V, u][W, g] } & =[V \otimes W, u g], \\
{[V, e]^{*} } & =\left[V^{*}, e\right], & {[\mathbf{1}, u]^{*} } & =[\mathbb{k}, u],
\end{aligned}
$$

$\alpha_{[V, u],[W, u],[Z, u]}=\left[ \pm \mathrm{id}_{V \otimes W \otimes Z}, u\right]$ and the others are trivial.

- The tensor product, dual objects and associativity in $\mathcal{D}(u, \iota, \pm 1)$ are given by

$$
\begin{aligned}
{[V, e][W, e] } & =[V \otimes W, e], & {[V, u][W, u] } & =\left[V \otimes W \otimes \mathbb{k}_{u}, e\right], \\
{[V, e][W, u] } & =[V \otimes W, u], & {[V, u][W, e] } & =[V \otimes W, u], \\
{[V, e]^{*} } & =\left[V^{*}, e\right], & {[\mathbf{1}, u]^{*} } & =\left[\mathbb{k}_{u}, u\right],
\end{aligned}
$$

$\alpha_{[V, u],[W, u],[Z, u]}=\left[ \pm \operatorname{id}_{V \otimes W} \otimes \tau\left(\varepsilon \iota \rho_{Z} \otimes \operatorname{id}_{Z \otimes \mathbb{k}_{u}}\right), u\right]$, where $\iota: H^{u} \rightarrow H$ is the unique bicomodule algebra isomorphism which satisfies $\iota(u)=-u$ and $\iota(x)=-x$ for $x \in V$; and $\tau: X \otimes Y \rightarrow Y \otimes X, \tau(z \otimes k)=k \otimes z$ for all $X, Y \in \operatorname{Comod}(H)$, see [8, Remark 2.2].

Remark 2.10. By Lemma 2.8(1), we obtain that only the categories $\mathcal{D}(1, \mathrm{id}, \pm 1)$ and $\mathcal{D}(u, \iota, \pm 1)$ could be braided, since the BiGalois objects have to be trivial.

By direct calculation on Equation (1), $\mathcal{D}(u, \iota,-1)$ is not braided with trivial braiding. So, in this case, we want to know if there exist another possible braidings. 


\section{Braided crossed product}

Let $\Gamma$ be an Abelian group. In [8], following the ideas developed in [3], we described all $\Gamma$-crossed product tensor categories which are extensions of $\operatorname{Comod}(H)$ for $H$ a Hopf algebra in terms of certain Hopf-algebraic datum. Fix $(H, r)$ a CQTHopf algebra. In the first Lemma of this Section, we do the same for the braiding of crossed products that are $\Gamma$-extensions of $\operatorname{Comod}(H)$.

Remark 3.1. If $v: H \rightarrow H$ is a left $H$-comodule morphism, since the coaction is the coproduct, $v$ satisfies $v(x)_{1} \otimes v(x)_{2}=x_{1} \otimes v\left(x_{2}\right)$, for all $x \in H$. In particular, $v$ is not a coalgebra morphism and if $g \in G(H), v(g)=g \varepsilon(v(g))$.

Lemma 3.2. Fix a datum $\left(g, f^{a, b}, \gamma\right)_{a, b \in \Gamma}$, as in Lemma 2.8, and let $\mathcal{C}$ be the associated tensor category. Consider a pair $\left(v^{a}, w^{a}\right)_{a \in \Gamma}$ where $v^{a}, w^{a}: H \rightarrow H$ are left $H$-comodule algebra isomorphisms. Let $W^{a}=\varepsilon w^{a}, V^{a}=\varepsilon v^{a}$ and $F^{a, b}=\varepsilon f^{a, b}$. If for all $a, b, c \in \Gamma$ and $X \in \operatorname{Comod}(H)$ we have

$$
\begin{gathered}
v^{1}=w^{1}=\operatorname{id}_{H}, \\
\left(g(a, b), f^{a, b}\right)=\left(g(b, a), f^{b, a}\right), \\
W^{b}\left(x_{-3}\right) W^{a}\left(x_{-2}\right)\left(W^{a b}\right)^{-1}\left(x_{-1}\right) x_{0}=F^{a, b}\left(x_{-2}\right) r\left(x_{-1} \otimes g(a, b)\right) x_{0}, \quad x \in X, \\
V^{b}\left(x_{-3}\right) V^{a}\left(x_{-2}\right)\left(V^{a b}\right)^{-1}\left(x_{-1}\right) x_{0}=r\left(x_{-2} \otimes g(a, b)\right) F^{a, b}\left(x_{-1}\right) x_{0}, \quad x \in X, \\
V^{a}(g(b, c))=\left(\gamma_{a, b, c} \gamma_{b, c, a}\right)^{-1} \gamma_{b, a, c}, \\
W^{b}(g(c, a))=\gamma_{c, a, b} \gamma_{b, c, a} \gamma_{c, b, a}^{-1} ;
\end{gathered}
$$

then we obtain a braiding over $\mathcal{C}$ given by

$\mathbf{c}_{[V, a],[W, b]}=c_{V, W}\left(\left(V^{a} \otimes \mathrm{id}\right) \rho_{V} \otimes\left(W^{a} \otimes \mathrm{id}\right) \rho_{W}\right) \otimes \mathrm{id}, \quad V, W \in \operatorname{Comod}(H), a, b \in \Gamma$.

All braidings over $\mathcal{C}$ come from a pair $\left(v^{a}, w^{a}\right)_{a \in \Gamma}$ which satisfies (7) to (11).

Proof. By [3, Definition 5.3], a datum $\left(g, f^{a, b}, \gamma\right)_{a, b \in \Gamma}$ has associated a braiding if there exist a triple $\left(\theta^{a}, \tau^{a}, t_{a, b}\right)_{a, b \in G}$ where

- $\theta^{a}, \tau^{a}: \mathrm{id}_{\mathcal{C}} \rightarrow \mathrm{id}_{\mathcal{C}}$ are monoidal natural isomorphisms,

- for all $a, b \in G, t_{a, b}:\left(U_{a, b}, \sigma^{a, b}\right) \rightarrow\left(U_{b, a}, \sigma^{b, a}\right)$ are isomorphisms in $\mathcal{Z}(\mathcal{C})$, where $\sigma_{X}^{a, b}=\tau\left(\varepsilon f^{a, b} \otimes \operatorname{id}_{X}\right) \rho_{X}$, for $\left(X, \rho_{X}\right) \in \operatorname{Comod}(H)$, and $U_{a, b}=$ $\mathbb{k}_{g(a, b)}$,

such that for all $a, b, c \in \Gamma$ and $X \in \mathcal{C}$, the following conditions hold

$$
\begin{gathered}
\theta^{1}=\tau^{1}=\mathrm{id}, \quad \theta_{\mathbf{1}}^{a}=\mathrm{id}_{\mathbf{1}}=\tau_{\mathbf{1}}^{a}, \quad t_{a, 1}=t_{1, a}=\mathrm{id}_{\mathbf{1}}, \\
c_{U_{a, b}, X} \sigma_{X}^{a, b}=\left(\left(\tau_{X}^{a b}\right)^{-1} \tau_{X}^{a} \tau_{X}^{b}\right) \otimes \mathrm{id}_{U_{a, b}},
\end{gathered}
$$




$$
\begin{gathered}
\sigma_{X}^{a, b} c_{U_{a, b}, X}=\operatorname{id}_{U_{a, b}} \otimes\left(\left(\theta_{X}^{a b}\right)^{-1} \theta_{X}^{a} \theta_{X}^{b}\right), \\
\gamma_{a, b, c}\left(\theta_{U_{b, c}}^{a} \otimes t_{b c, a}\right) \gamma_{b, c, a}=\left(t_{b, c} \otimes \operatorname{id}_{U_{b a, c}}\right) \gamma_{b, a, c}\left(t_{c, a} \otimes \operatorname{id}_{U_{b, a c}}\right), \\
\gamma_{c, a, b}^{-1}\left(\tau_{c, a}^{b} \otimes t_{b, c a}\right) \gamma_{b, c, a}^{-1}=\left(t_{b, a} \otimes \operatorname{id}_{U_{c, b a}}\right) \gamma_{c, b, a}^{-1}\left(t_{b, c} \otimes \operatorname{id}_{U_{b c, a}}\right) .
\end{gathered}
$$

By Lemma 2.1, each monoidal natural isomorphism of the identity functor comes from a left $H$-comodule algebra isomorphism, then $\theta_{X}^{a}:=\left(\varepsilon v^{a} \otimes \mathrm{id}\right) \rho_{X}$ and $\tau_{X}^{a}:=$ $\left(\varepsilon w^{a} \otimes \operatorname{id}_{X}\right) \rho_{X}$ for all $X \in \operatorname{Comod}(H)$. Since $U_{g(a, b)}=\mathbb{k}_{g(a, b)}$, we can take $t_{a, b} \in \mathbb{k}^{*}$.

Each $t_{a, b}$ is a left $H$-comodule isomorphism if and only if $g(a, b) \otimes t_{a, b} \mathrm{id}_{\mathbb{k}}=$ $g(b, a) \otimes t_{a, b} \operatorname{id}_{\mathbb{k}}$ which gives $g(a, b)=g(b, a)$ for all $a, b \in \Gamma$. Moreover, each $t_{a, b}$ is a braided morphism if and only if $\sigma_{X}^{a, b} t_{a, b}=\sigma_{X}^{b, a} t_{a, b}$ for $a, b \in \Gamma$ and $X \in \operatorname{Comod}(H)$ if and only if $\sigma^{a, b}=\sigma^{b, a}$. Then $t_{a, b}$ is an isomorphism in $\mathcal{Z}(\operatorname{Comod}(H))$ if and only if Condition (7) holds.

Condition (12) is equivalent to $v^{1}=w^{1}=\operatorname{id}_{H}$ and $t_{a, 1}=t_{1, a}=1$, since $\theta_{\mathrm{k}}^{a}=\mathrm{id}_{\mathbb{k}}=\tau_{\mathrm{k}}^{a}$ is always true. Condition (13) is equivalent to

$$
F^{a, b}\left(x_{-2}\right) r\left(x_{-1} \otimes g(a, b)\right) x_{0} \otimes k=W^{b}\left(x_{-3}\right) W^{a}\left(x_{-2}\right)\left(W^{a b}\right)^{-1}\left(x_{-1}\right) x_{0} \otimes k,
$$

for $x \otimes k \in X \otimes \mathbb{k}_{g(a, b)}$, which is equivalent to Condition (8). In the same way, Condition (14) is equivalent to Condition (9). Condition (15) is equivalent to $\gamma_{a, b, c} V^{a}(g(b, c)) t_{b c, a} \gamma_{b, c, a}=t_{b, c} \gamma_{b, a, c} t_{c, a}$ but if we take $c=1$ then

$$
1=t_{b, a}, \text { for } a, b \in \Gamma,
$$

so, this Condition is equivalent to Condition (10), and Condition (16) is equivalent to Condition (11).

By $[3$, Theorem 5.4], this pair produces a braiding over $\mathcal{C}$ given by

$$
\mathbf{c}_{[V, a],[W, b]}=\left[c_{V, W}\left(\theta_{V}^{a} \otimes \tau_{W}^{a}\right), a b\right], \quad \text { for all } V, W \in \operatorname{Comod}(H), a, b \in \Gamma,
$$

and all braidings come from such a pair.

Now, we focus our attention into the case $\Gamma=C_{2}$. By Lemma 2.8, a datum $\Upsilon^{\prime}=$ $(g, f, \gamma)$ with $g \in G(H)$ a group-like element, $f: H^{g} \rightarrow H$ a bicomodule algebra isomorphism and $\gamma \in \mathbb{k}^{\times}, \gamma^{2}=1$; generates a tensor category $\mathcal{C}=\operatorname{Comod}(H)\left(\Upsilon^{\prime}\right)$.

The following theorem gives us the third and last condition to decide if our categories are braidable.

Theorem 3.3. The category $\operatorname{Comod}(H)\left(\Upsilon^{\prime}\right)$ is a braided $C_{2}$-extension if and only if, there exists a pair of isomorphisms of left $H$-comodule algebras $v, w: H \rightarrow H$ such that for all $X \in \operatorname{Comod} H$ and $x \in X$

a. $\varepsilon\left(w\left(x_{-2}\right) w^{-1}\left(x_{-1}\right)\right) x_{0}=x$, 
b. $\varepsilon\left(w\left(x_{-2}\right) w\left(x_{-1}\right)\right) x_{0}=\varepsilon f\left(x_{-2}\right) r\left(x_{-1} \otimes g\right) x_{0}$,

c. $\varepsilon\left(v\left(x_{-2}\right) v^{-1}\left(x_{-1}\right)\right) x_{0}=x$,

d. $\varepsilon\left(v\left(x_{-2}\right) v\left(x_{-1}\right)\right) x_{0}=r\left(x_{-2} \otimes g\right) \varepsilon f\left(x_{-1}\right) x_{0}$,

e. $\varepsilon(v(g))=\gamma^{-1}$,

f. $\varepsilon(w(g))=\gamma$.

Proof. Condition (7) is always true. Condition (8) is equivalent to $r\left(x_{-1} \otimes 1\right) x_{0}=$ $x$, and items a,b. Condition (9) is equivalent to $r\left(x_{-1} \otimes 1\right) x_{0}=x$, and items c,d. Condition (10) is equivalent to item e. Condition (11) is equivalent to item $\mathrm{f}$.

Regarding condition $r\left(x_{-1} \otimes 1\right) x_{0}=x$, it is always true over a CoQuasi-triangular Hopf algebra.

If $H=\wedge V \# \mathbb{k} C_{2}$, as Example 2.4, by [8, Proposition 4.10], the isomorphisms $v$ and $w$ are identities. Then if the extension is braided the only possible braiding is the trivial, see Equation (17), since the category Comod $H$ has a braiding giving by the r-form. With this information, Conditions a-f are equivalent to

$$
\begin{aligned}
& \text { a'. } \varepsilon\left(x_{-2} x_{-1}\right) x_{0}=x, \\
& \text { b'. } \varepsilon\left(x_{-2} x_{-1}\right) x_{0}=\varepsilon f\left(x_{-2}\right) r\left(x_{-1} \otimes g\right) x_{0}, \\
& \text { c'. } \varepsilon\left(x_{-2} x_{-1}\right) x_{0}=r\left(x_{-2} \otimes g\right) \varepsilon f\left(x_{-1}\right) x_{0}, \\
& \text { d'. } \varepsilon(g)=\gamma^{-1}, \\
& \text { e'. } \varepsilon(g)=\gamma .
\end{aligned}
$$

Since $g$ is a group-like element, d' and e' imply that $\gamma=1$. Thus, the only categories that could be braided are $\mathcal{D}(1, \mathrm{id}, 1)$ and $\mathcal{D}(u, \iota, 1)$.

Corollary 3.4. A $C_{2}$-extension over $\operatorname{Comod}\left(\wedge V \# \mathbb{k} C_{2}\right)$ is braided if and only if, for all comodule $X, r\left(f\left(x_{-1}\right) \otimes g\right) x_{0}=x$, for all $x \in X$.

Proof. Condition a' is always true over comodules. Since $x_{1} y_{1} r\left(x_{2} \otimes y_{2}\right)=r\left(x_{1} \otimes\right.$ $\left.y_{1}\right) y_{2} x_{2}$ for $x, y \in H$ we have

$$
\left(x_{-1} g\right) r\left(x_{-2} \otimes g\right) \otimes x_{0}=r\left(x_{-1} \otimes g\right) g x_{-2} \otimes x_{0} .
$$

Applying $\varepsilon f \otimes \mathrm{id}_{X}$, we obtain $r\left(x_{-2} \otimes g\right) \varepsilon f\left(x_{-1}\right) x_{0}=\varepsilon f\left(x_{-2}\right) r\left(x_{-1} \otimes g\right) x_{0}$. This implies that Conditions b' and c' are equivalent. Since

$$
r(f(x) \otimes g)=r\left(f(x)_{1} \otimes g\right) \varepsilon\left(g\left(f(x)_{2}\right)\right)=r\left(x_{1} \otimes g\right) \varepsilon\left(f\left(x_{2}\right)\right)
$$

we have $r\left(f\left(x_{-1}\right) \otimes g\right) x_{0}=\varepsilon f\left(x_{-2}\right) r\left(x_{-1} \otimes g\right) x_{0}$, then Condition b' is equivalent to

$$
r\left(f\left(x_{-1}\right) \otimes g\right) x_{0}=x .
$$

We are ready for our main result. 
Theorem 3.5. The categories $\mathcal{D}(1, \mathrm{id}, 1)$ and $\mathcal{D}(u, \iota, 1)$ are braided tensor categories. The remaining 6 categories found in [8] are non-braidable.

Proof. By [3, Theorem 5.4], the only possible option for $v$ and $w$ is for there to be the identity. Then the categories $\mathcal{D}(1, \mathrm{id}, 1)$ and $\mathcal{D}(u, \iota, 1)$ have associated at most a single pair (id, id), which would give it a braided structure For the remaining six categories, we already know that they are non-braidable.

Since $\mathcal{D}(1, \mathrm{id}, 1)$ has trivial associativity and $\operatorname{Comod}(H)$ is braided then the braiding for $\mathcal{D}(1, \mathrm{id}, 1)$ is

$$
\mathbf{c}_{[V, a],[W, b]}=\left[c_{V, W}, a b\right], \quad \text { for all } V, W \in \operatorname{Comod}(H), a, b \in C_{2} .
$$

Over $\mathcal{D}(u, \iota, 1)$ it is enough to check Equations (1) and (2) where the associativity is not trivial. Since $(f \otimes \mathrm{id})(\mathrm{id} \otimes g)=(\mathrm{id} \otimes g)(f \otimes \mathrm{id})$ for any $f, g$ morphisms in the category, also the braiding given in (18) also satisfies the desired Equations.

Corollary 3.6. For $X \in \operatorname{Comod}\left(\wedge V \# \mathbb{k} C_{2}\right), r\left(\iota\left(x_{-1}\right) \otimes g\right) x_{0}=x$, for all $x \in X$.

Remark 3.7. Since $\operatorname{Comod}(H)$ is not symmetric, then these two categories are not symmetric either.

\section{References}

[1] N. Andruskiewitsch and M. Grana, Braided Hopf algebras over non abelian finite groups, Colloquium on Operator Algebras and Quantum Groups (Spanish) (Vaquerias, 1997), Bol. Acad. Nac. Cienc. (Cordoba), 63 (1999), 45-78.

[2] P. Etingof and S. Gelaki, The classification of finite-dimensional triangular Hopf algebras over an algebraically closed field of characteristic 0, Mosc. Math. J., 3(1) (2003), 37-43.

[3] C. Galindo, Crossed product tensor categories, J. Algebra, 337 (2011), 233-252.

[4] T. J. Hagge and S.-M. Hong, Some non-braided fusion categories of rank three, Commun. Contemp. Math., 11(4) (2009), 615-637.

[5] A. Joyal and R. Street, Braided Monoidal Categories, Macquarie Math. Reports, Report No: 860081, November 1986.

[6] A. Masuoka, Cocycle deformations and Galois objects for some cosemisimple Hopf algebras of finite dimension, in New trends in Hopf algebra theory (La Falda, 1999), Contemp. Math., 267 (2000), 195-214.

[7] A. Masuoka, Example of almost commutative Hopf algebras which are not coquasitriangular, Hopf algebras, Lecture Notes in Pure and Appl. Math., Dekker, New York, 237 (2004), 185-191. 
[8] A. Mejia Castano and M. Mombelli, Crossed extensions of the corepresentation category of finite supergroup algebras, Internat. J. Math., 26(9) (2015), 1550067 (26 pp).

[9] S. Montgomery, Hopf Algebras and Their Actions on Rings, CBMS Regional Conference Series in Mathematics, 82, Published for the Conference Board of the Mathematical Sciences, Washington, DC; by the Amer. Math. Soc., Providence, RI, 1993.

[10] P. Schauenburg, Hopf bi-Galois extensions, Comm. Algebra, 24(12) (1996), 3797-3825.

\author{
Mejía Castaño Adriana \\ Departamento de Matemáticas y Estadística \\ Facultad de Ciencias Básicas \\ Universidad del Norte \\ Barranquilla, Colombia \\ e-mails: sighana25@gmail.com, mejiala@uninorte.edu.co
}

